\title{
All the questions about biophysics
}

\author{
Harness the hubris: useful things physicists could do in biology \\ Authors: V. Adrian Parsegian and Commentary by Robert H. Austin \\ Physics Today 50, 7, 23 (1997) \\ Recommended with a Commentary by Ramin Golestanian, Max \\ Planck Institute for Dynamics and Self-Organization \\ (Göttingen) and University of Oxford
}

What is biophysics? This was one of the most important questions on my mind in 1997, when I was approaching the end of my $\mathrm{PhD}$ and thinking about planning my future academic career (by looking for a postdoctoral research position). I had performed some theoretical work on physical questions that concern biological systems and I was trying to find out what that sort of work makes me, and whether I can pursue this style of research for a career. I wrote to a number of people whom I knew from their works (asking for a postdoc position) and some of them proposed to talk to me during an upcoming major international meeting. What I gathered from these discussions were strongly aligned with the spirit of the "Parsegian-Austin" debate [1], which essentially poses the question we started with (if we make our way through the oversimplified stereotyping arguments). After more than two decades, the topic is still as relevant as ever, and I thought it would be important to reflect on it for the benefit of the future generations [2].

Naturally, the discussion today needs to be more comprehensive and nuanced, and include a much wider range of questions. What does it mean to have an impact in biophysics? Who is a biophysicist? How can I evaluate a biophysicist colleague? What type of research agenda should be considered as high-quality biophysics work? There are many questions like these that many of us are grappling with. We could be very early career aspiring researchers and trying to plan our future, early career researchers and trying to steer our plans through the rugged academic landscapes, or established researchers in the field and struggling to explain ourselves to others. Or we could be senior researchers serving on evaluation panels for grants and promotions, advisory boards for conferences, and search committees for academic hires, editors of high-profile journals and trying to decide what type of work should be considered sufficiently important for consideration, or even decision-makers in national science funding agencies and needing to decide how to allocate funds for interdisciplinary research. We would all face these questions.

An important way to define and delineate the identities of different groups of people at the interface between established fields of science is through terminology. For example, the terms chemical physics and physical chemistry have been used in many countries (but not all) to emphasize the difference between those who identify as physicists interested in chemical 
research topics and vice versa. For biophysics, a number of different variants have been used, albeit less successfully: biophysics, biological physics, biologically-inspired physics, physical biology, and so on. The website of the (American) Biophysical Society offers the following definition: "Biophysics is the field that applies the theories and methods of physics to understand how biological systems work" [3]. Although this might at first sight appear to be a sufficiently clear definition, it suffers from a significant ambiguity because it does not specify what it means to "understand" something. Understanding will mean different things to different people. What the definition is very clear about, however, is that the "theories and methods" will come from physics. One can argue that this definition may have originated from the dominant point of view of the biologists, but I do not wish to engage with the 'harness the hubris debate' here. I want to propose a fresh perspective.

The interface between physics and biology offers one of the most exciting research opportunities for the $21^{\text {st }}$ century; it covers questions ranging from the origin of life to understanding why living systems are hierarchically structured and how they can achieve homeostasis under non-equilibrium conditions. These challenges will keep physicists busy for many years to come. Therefore, it is important to find a way to answer the above questions, as we cannot afford to get them wrong.

The first step towards answering the questions is to acknowledge that the interface between physics and biology offers a wide spectrum of possibilities, and it will be naturally wrong of us to assume that there is only one type of biophysics or biophysicist. I have found over the years that a good way to break down this wide spectrum is to use the following four indices for researchers in biophysics: the background training they have had, the tools and techniques that they use, the systems they are studying, and the sort of questions they are trying to answer. A combinatorial construction with these indices is shown in the Table below. Viewed in this way, it is evident that there are many different flavours of biophysics that cannot be directly compared with one another. They can, of course, have very powerful synergistic relationships.

There are many physicists who choose Class 8 for their career and immigrate to biology, or choose Class 4 and take powerful tools with them in addition to their training. Due to the nature of this path, the right way to evaluate the research outcome of such physicists is to gauge the impact they have had in biology by asking the opinion of biologists. For those who choose Class 3, on the other hand, the impact should be gauged by probing the contribution they make towards developing new physical insights into the complex behaviour of nonequilibrium living matter. Evidently, it would be a travesty to evaluate Class 3 based on the appropriate criteria for Class 8 or Class 4 , and vice versa. Other cases can also be considered to explore the different styles of biophysical research that are possible. Interestingly, there are some combinations that offer new challenges, and can be considered for future expansions of the research agenda at this interface, even though at the moment we might not have that many existing examples in such categories, e.g. Classes 13, 14, and 15.

These indices can play an important role in building research communities. Similar training provides a common language that is essential for community cohesion, and tool development is a major part of the work for most researchers and they will find it helpful to share tips and technical expertise. The use of common systems allows researchers to compile practical knowledge about calibration, sample preparation, and so on, whereas common questions give focus to shared research programmes. 


\begin{tabular}{|c|c|c|c|c|c|}
\hline \multicolumn{2}{|c|}{ training } & tools & systems & questions & comment \\
\hline 1 & phys & phys & phys & phys & physics \\
\hline 2 & phys & phys & phys & bio & synthetic living systems; origin of life \\
\hline 3 & phys & phys & bio & phys & biological systems as living matter \\
\hline 4 & phys & phys & bio & bio & bio-oriented biophysics; instrumentation \\
\hline 5 & phys & bio & phys & phys & a new challenge? \\
\hline 6 & phys & bio & phys & bio & a new challenge? \\
\hline 7 & phys & bio & bio & phys & a new challenge? \\
\hline 8 & phys & bio & bio & bio & (immigration to) biology \\
\hline 9 & bio & phys & phys & phys & (immigration to) physics \\
\hline 10 & bio & phys & phys & bio & synthetic living systems; origin of life \\
\hline 11 & bio & phys & bio & phys & biological systems as living matter \\
\hline 12 & bio & phys & bio & bio & bio-oriented biophysics; biology \\
\hline 13 & bio & bio & phys & phys & a new challenge? \\
\hline 14 & bio & bio & phys & bio & a new challenge? \\
\hline 15 & bio & bio & bio & phys & a new challenge? \\
\hline 16 & bio & bio & bio & bio & biology \\
\hline
\end{tabular}

Figure 1: A combinatorial table classifying different variants of research style at the interface between physics (phys) and biology (bio).

The interdisciplinarity of the field allows people to complement each other, which means that many research teams are defined based on the last two indices, i.e. systems and questions. They typically contain members that cover complementary realizations of the first two indices, i.e. training and tools. Moreover, in recent years a number of interdisciplinary training programmes have been established to develop background that can cover physics and biology both in terms of academic training and techniques. While such plans to integrate interdisciplinarity at the early stages of training will help blur some of the divisions arising from the classification shown in the Table, I believe awareness of the existence of such differences is important when we try to define what biophysics is, and find answers to all the questions about biophysics.

\section{References}

[1] V. Adrian Parsegian, Harness the hubris: useful things physicists could do in biology, Physics Today 50, 7, 23 (1997); see also the Commentary by Robert H. Austin to this article, and the accompanying reactions: How should physicists, biologists work together? The 'harness the hubris' debate continues, Physics Today 50, 12, 11 (1997). 
[2] This commentary is a slightly modified version of an essay that I wrote for the Newsletter of the IUPAP Commission on Biological Physics (C6) in September 2019.

[3] What Is Biophysics from the website of the Biophysical Society. 